\title{
Introducing two hyperparameters in Bayesian estimation of wave spectra
}

\author{
Nielsen, Ulrik Dam
}

Published in:

Probabilistic Engineering Mechanics

Link to article, DOI:

10.1016/j.probengmech.2007.10.007

Publication date:

2008

Link back to DTU Orbit

Citation (APA):

Nielsen, U. D. (2008). Introducing two hyperparameters in Bayesian estimation of wave spectra. Probabilistic Engineering Mechanics, 23(1), 84-94. https://doi.org/10.1016/j.probengmech.2007.10.007

\section{General rights}

Copyright and moral rights for the publications made accessible in the public portal are retained by the authors and/or other copyright owners and it is a condition of accessing publications that users recognise and abide by the legal requirements associated with these rights.

- Users may download and print one copy of any publication from the public portal for the purpose of private study or research.

- You may not further distribute the material or use it for any profit-making activity or commercial gain

- You may freely distribute the URL identifying the publication in the public portal

If you believe that this document breaches copyright please contact us providing details, and we will remove access to the work immediately and investigate your claim. 


\title{
Introducing Two Hyperparameters in Bayesian Estimation of Wave Spectra
}

\author{
ULRIK DAM NIELSEN* \\ Department of Mechanical Engineering, \\ Technical University of Denmark, DK-2800 Kgs. Lyngby, Denmark
}

\begin{abstract}
An estimate of the on-site wave spectrum can be obtained from measured ship responses by use of Bayesian modelling, which means that the wave spectrum is found as the optimum solution from a probabilistic viewpoint. The paper describes the introduction of two hyperparameters into Bayesian modelling so that the prior information included in the modelling is based on two constraints: the wave spectrum must be smooth directional-wise as well as frequency-wise. Traditionally, only one hyperparameter has been used to control the amount of smoothing applied in both the frequency and directional ranges. From numerical simulations of stochastic response measurements, it is shown that the optimal hyperparameters, determined by use of ABIC (a Bayesian Information Criterion), correspond to the best estimate of the wave spectrum, which is not always the case when only one hyperparameter is included in the Bayesian modelling. The paper includes also an analysis of full-scale motion measurements where wave spectra estimated by the Bayesian modelling are compared with results from ocean surface measurements by satellite and from a wave radar. The agreement is found to be reasonable.
\end{abstract}

Key words: Wave spectra, measured ship responses, probabilistic viewpoint, Bayesian modelling, ABIC, two hyperparameters, stochastic simulations, full-scale measurements

\section{INTRODUCTION}

1.1. Background and Motivation. Navigational and operational guidance to the master of a ship can be given by use of in-service monitoring, or decision support, systems where a set of sensors measures specific ship responses. The topic is widely dealt with in the literature, e.g Huss and Olander [12], Debord and Hennessy [5] and Slaughter et al. [26], and as well by different kinds of commercial and academia projects, e.g. Nielsen et al. [18] and the EU project ADOPT [1]. For the given guidance to be reliable it is paramount to have information, in the statistical sense, about

*E-mail: udn@mek.dtu.dk $\quad$ Ph: +45 45251970 
the wave excitations of the ship. This information can be given in terms of the on-site (directional) wave spectrum.

On-site wave spectra can be estimated on the basis of measured ship responses (e.g. motion data) by use of Bayesian modelling, c.f. Iseki and Terada [14] and Nielsen [20]. Thus, in addition to the physical equations relating the measured and the calculated responses, prior constraints are introduced to make the wave spectrum smooth. The relative weight of the applied smoothness is governed by a so-called hyperparameter. Basically, this means that Bayesian modelling is a tradeoff between the good-fit of the solution to the data (i.e. agreement between data and solution) and the smoothness, or stability, of the solution. Therefore, an optimal value of the hyperparameter must be considered, and the criterion, which is normally used for the selection, is ABIC (a Bayesian Information Criterion), see Akaike [2]. In this sense, the on-site wave spectrum is found as the optimal solution from a probabilistic viewpoint.

Typically, the wave spectrum is assumed to be smooth in the frequency and the directional ranges with only one hyperparameter to control the amount of smoothness, e.g. Iseki and Ohtsu [13]. The study of data and associated estimated (and measured) wave spectra, e.g. Nielsen [20], reveals, however, that the use of one optimal hyperparameter does often not give realistic estimations of the wave spectra. The reason is that two different hyperparameters must be considered in order to properly control the amount of smoothing in both the directional range and the frequency range.

In view of decision support systems for safe navigation of ships, it is important to rely on an automatically selection of the best estimation of the on-site wave spectrum. Since problems in this respect, thus, have been encountered, when only one hyperparameter is considered in the Bayesian modelling, it is of interest to develop the Bayesian modelling so that it facilitates an automatically selection of the (best) wave spectrum.

1.2. The Study. This paper addresses the introduction of two hyperparameters in the Bayesian modelling and, thus, ABIC must be given in a form that takes two hyperparameters into account. The introduction of two hyperparameters is similar to the geophysical description of geodetic data inversion to estimate slip history at a plate interface based on viscoelastic slip-response functions. Hence, Fukahata et al. [8] and Fukahata et al. [9] introduced a proper formulation of ABIC to incorporate two sorts of partially dependent prior information into observed data.

The paper contains a study of numerical simulations of time histories with focus on the estimation of wave spectra. Thus, the wave estimations are carried out from numerical simulations with exact 
known underlying wave excitations, i.e. wave parameters. In this way it is possible to evaluate that the minimum value of $\mathrm{ABIC}$ does correspond to the optimal values of hyperparameters in the sense that the best solution, as regards the good-fit as well as stability of the solution, is obtained for the specific selection, by ABIC, of hyperparameters. This is in contrast to the case when only one hyperparameter is considered in the Bayesian modelling, which will be illustrated by examples.

1.3. Composition of Paper. The organisation of the paper is as follows. In Section 2, the fundamental theory of Bayesian modelling is outlined. In addition, Section 2 gives the details on how to include two hyperparameters in Bayesian modelling with a particular focus on formulating ABIC properly. The calculation of specific sea state parameters is described in Section 3. Section 4 deals with examples based on numerical simulations of motion measurements of ship responses, whereas Section 5 contains the analysis of full-scale measurements. In both sections the results are primarily given in tabular form and visualised graphically. Finally, Section 6 summarises and concludes on the presented material.

\section{BAyesian Modelling}

2.1. Fundamentals. The present subsection gives the fundamentals of Bayesian modelling applied to estimate directional wave spectra. The subsection is by no means comprehensive and for details the literature should be consulted, e.g. Iseki and Terada [14] and Nielsen [20].

On the assumption that the ship responses are stationary and linear with the incident waves, the complex-valued frequency response functions $\Phi_{i}\left(\omega_{e}, \beta\right)$ and $\Phi_{j}\left(\omega_{e}, \beta\right)$ for the $i$ th and $j$ th responses yield the theoretical relationship between the $i$ th and the $j$ th components of the cross spectra $S_{i j}\left(\omega_{e}\right)$ and the directional wave spectrum $E\left(\omega_{e}, \beta\right)$ through the following integral equation

$$
S_{i j}\left(\omega_{e}\right)=\int_{-\pi}^{\pi} \Phi_{i}\left(\omega_{e}, \beta\right) \overline{\Phi_{j}\left(\omega_{e}, \beta\right)} E\left(\omega_{e}, \beta\right) d \beta
$$

where the bar denotes the complex conjugate, and with $\beta$ being the heading of the ship (relative to the waves) and $\omega_{e}$ being the encounter frequency. The heading is defined so that $\beta=\pi$ corresponds to head waves. It should be realised that the wave spectrum is given in terms of the heading, which is justified by letting the wave direction, $\theta$, and the course of the ship be given relative to the same datum, so that the heading and the wave direction are coincident. Finally, it should be noted that the complex-valued frequency response functions are written as functions of only the heading and the encounter frequency, since the implication of changing operational parameters is understood. 
The wave spectrum is advantageously estimated in the wave frequency domain. This means that the speed-of-advance, or triple-valued function, problem needs to be considered. The triple-valued function problem stems from the relation/requirement

$$
\omega_{e}=\omega-\omega^{2} A \quad, \quad A=\frac{V}{g} \cos \beta
$$

where $\omega$ is the true wave frequency, $V$ is the speed of the ship, and $g$ is the acceleration of gravity. The requirement has been properly incorporated by Iseki and Ohtsu [13], for details see Nielsen $[20]$.

In terms of matrix notation, Eq. (1) can be written

$$
\mathbf{b}=\mathbf{A f}(\mathbf{x})+\mathbf{w}
$$

The vector function $\mathbf{f}(\mathbf{x})$ expresses the unknown values of the wave spectrum $E(\omega, \beta)$ through a non-negativity constraint $\mathbf{f}(\mathbf{x})=\exp (\mathbf{x})$, so that $\mathbf{x}=\ln E(\omega, \beta)$. w is a Gaussian white noise sequence vector with zero mean and variance $\sigma^{2}$, introduced for stochastic reasons so that the Bayesian modelling is facilitated, cf. Akaike [2]. The vector $\mathbf{b}$ contains the elements of $S_{i j}\left(\omega_{e}\right)$, and the coefficient matrix $\mathbf{A}$ has elements according to the products of the transfer functions and the derivative of the wave frequency, cf. Eq. (1).

In principle, Eq. (3) can be solved for $\mathbf{x}$ by minimising $\chi^{2}(\mathbf{x})$ with

$$
\chi^{2}(\mathbf{x}) \equiv\|\mathbf{A f}(\mathbf{x})-\mathbf{b}\|^{2}
$$

where $\|\cdot\|$ represents the $L_{2}$ norm.

2.2. Prior Information. The equation system given by Eq. (4) is in most cases underdetermined, or otherwise degenerate, which means that the solution is unstable. To overcome this problem Bayesian modelling is introduced, see Akaike [2], so that $\mathbf{x}$ is evaluated by maximisation of the product of the likelihood function and the prior distributions, which must be defined properly. The prior distributions act as a stochastic constraint and are a general character of the model that is known in advance, e.g. Iseki and Terada [14]. For the model above, the likelihood function is written as

$$
l\left(\mathbf{x} \mid \sigma^{2}\right)=\left(\frac{1}{2 \pi \sigma^{2}}\right)^{\frac{P}{2}} \exp \left(-\frac{1}{2 \sigma^{2}}\|\mathbf{A f}(\mathbf{x})-\mathbf{b}\|^{2}\right)
$$

where $P$ is the total number of integral equations derived from Eq. (1) including a number of equations yielding equivalence of energy on the left- and right-hand side of Eq. (1), cf. Nielsen [20]. 
In this paper, two prior distributions are taken into account. Both distributions are a Gaussian smoothness prior distribution which minimises the sum of the second order difference of the unknown vector $\mathbf{x}$ in order to smoothen the change with frequency and direction, respectively, of the wave spectrum, e.g. Nielsen [20] and Iseki and Terada [14]. The prior distributions are therefore defined by the minimisation of the functionals

$$
\begin{aligned}
\sum_{n=1}^{N} \sum_{m=1}^{M} \varepsilon_{1 m n}^{2}= & \sum_{n=1}^{N} \sum_{m=1}^{M}\left(x_{m-1, n}-2 x_{m, n}+x_{m+1, n}\right)^{2} ; \quad\left(x_{0, n}=x_{M, n}, x_{M+1, n}=x_{1, n}\right) \\
& \sum_{n=1}^{N} \sum_{m=1}^{M} \varepsilon_{2 m n}^{2}=\sum_{m=1}^{M} \sum_{n=2}^{N-1}\left(x_{m, n-1}-2 x_{m, n}+x_{m, n+1}\right)^{2}
\end{aligned}
$$

where $N$ and $M$ are the number of discrete wave frequencies and discrete headings, respectively. Thus, considering $\varepsilon_{1 m n}$ and $\varepsilon_{2 m n}$ to be normal distributions with zero mean and variance $\sigma^{2} / u^{2}$ and $\sigma^{2} / v^{2}$, respectively, the prior distribution is given in terms of the so-called hyperparameters $u$ and $v$. In matrix notation the functionals can be written, see e.g. Press et al. [24]

$$
\begin{aligned}
& \sum_{n=1}^{N} \sum_{m=1}^{M} \varepsilon_{1 m n}^{2}=\mathbf{x}^{T} \mathbf{H}_{1} \mathbf{x} \\
& \sum_{n=1}^{N} \sum_{m=1}^{M} \varepsilon_{2 m n}^{2}=\mathbf{x}^{T} \mathbf{H}_{2} \mathbf{x}
\end{aligned}
$$

In accordance with Akaike [2], the posterior distribution $p\left(\mathbf{x} \mid u, v, \sigma^{2}\right)$ is proportional to the product of the likelihood function and the prior distribution, which can be written, cf. Fukahata et al. [8],

$$
p\left(\mathbf{x} \mid u, v, \sigma^{2}\right)=c\left(\frac{1}{2 \pi \sigma^{2}}\right)^{\frac{P+K M}{2}}\left|\operatorname{det}\left(u^{2} \mathbf{H}_{1}+v^{2} \mathbf{H}_{2}\right)\right|^{1 / 2} \exp \left(-\frac{1}{2 \sigma^{2}} S(\mathbf{x})\right)
$$

with

$$
S(\mathbf{x})=\|\mathbf{A f}(\mathbf{x})-\mathbf{b}\|^{2}+\mathbf{x}^{T}\left(u^{2} \mathbf{H}_{1}+v^{2} \mathbf{H}_{2}\right) \mathbf{x}
$$

and where $c$ is a normalising factor independent of the model parameters $\mathbf{x}$ and the hyperparameters $u$ and $v$. Akaike [2] proposes to maximise the posterior distribution for the evaluation of $\mathbf{x}$, which equivalently corresponds to minimising $S(\mathbf{x})$. In this regard it is necessary to linearise the nonnegativity constraint $\mathbf{f}(\mathbf{x})$; see Nielsen [19].

The hyperparameters control the trade-off between the good-fit of the solution to the data and the smoothness, or stability, of the solution, and the optimum values of the hyperparameters are determined by minimising the control criterion ABIC, cf. Akaike [2],

$$
\mathrm{ABIC}=-2 \ln \int p\left(\mathbf{x} \mid u, v, \sigma^{2}\right) d \mathbf{x}
$$


i.e. the minimisation of minus twice the logarithm to the marginal likelihood of the parameter(s) which may be in question. In general, such a minimisation of a likelihood calls for an approximation due to the high-dimensional integral. This matter is an entire subject of its own and is treated among many others by Davison [4] and Shun and McCullagh [25]. The calculation of the integral in Eq. (12) yields the following expression

$$
\mathrm{ABIC}=P \ln S\left(\mathbf{x}^{*}\right)-\ln \left|\operatorname{det}\left(u^{2} \mathbf{H}_{1}+v^{2} \mathbf{H}_{2}\right)\right|+\ln \left|\operatorname{det}\left(\mathbf{A}^{T} \mathbf{A}+u^{2} \mathbf{H}_{1}+v^{2} \mathbf{H}_{2}\right)\right|+C
$$

where the best estimate of $\mathbf{x}=\mathbf{x}^{*}$ for fixed $u^{2}$ and $v^{2}$ can be obtained by

$$
S\left(\mathbf{x}^{*}\right)=P \cdot \sigma_{\min }^{2}
$$

Here, $\sigma_{\min }^{2}$ is the minimum variance of the residuals in the minimisation of $S(\mathbf{x})$. The integration constant $C$ is independent of the hyperparameters $u$ and $v$, and the search for the values of $u$ and $v$ which minimise ABIC can be carried out numerically. Hence, with the values of the hyperparameters minimising ABIC, the best estimate of $\mathbf{x}=\mathbf{x}^{*}$ are - schematically - obtained from, cf. Press et al. $[24]$

$$
\mathbf{x}^{*}=\left(\frac{1}{\mathbf{A}^{T} \mathbf{A}+u^{2} \mathbf{H}_{1}+v^{2} \mathbf{H}_{2}} \mathbf{A}^{T} \mathbf{A}\right) \mathbf{A}^{-1} \mathbf{b} \quad \text { (schematic only!) }
$$

Basically, this finishes the Bayesian modelling, although with many details on algebra and numeric left out. Details can be found in Nielsen [20] and Nielsen [19].

\section{Integrated Wave Parameters}

The overall outcome of the Bayesian modelling is given by a directional wave spectrum $E(\omega, \theta)$. For comparative reasons, integrated wave parameters need therefore to be evaluated in each estimation, and this subsection serves as the reference for the calculation of these parameters.

The frequency wave spectrum is obtained by integrating the directional wave spectrum with respect to direction

$$
F(\omega)=\int_{-\pi}^{\pi} E(\omega, \theta) d \theta
$$

and hence the spectral moment of order $n$ is defined by

$$
m_{n}=\int_{0}^{\infty} \omega^{n} F(\omega) d \omega
$$

where the latter integral, in practical computations, extends from zero to a sufficiently large value of $\omega$. 
Thus, the significant wave height $H_{s}$ and the mean wave period $T_{s}$ are estimated from

$$
\begin{aligned}
& H_{s}=4 \sqrt{m_{0}} \\
& T_{s}=2 \pi \frac{m_{0}}{m_{1}}
\end{aligned}
$$

In addition, the peak period is

$$
T_{p}=\frac{2 \pi}{\omega_{p}}, \quad F\left(\omega_{p}\right)=\max _{\omega} F(\omega)
$$

The mean heading $\beta_{s}$ is derived on the basis of the course of the vessel in combination with the mean wave direction given by

$$
\theta_{s}=\arctan (d / c)
$$

where $d$ and $c$ are defined according to

$$
\begin{aligned}
& d=\int_{-\pi}^{\pi} \int_{0}^{\infty} E(\omega, \theta) \sin \theta d \omega d \theta \\
& c=\int_{-\pi}^{\pi} \int_{0}^{\infty} E(\omega, \theta) \cos \theta d \omega d \theta
\end{aligned}
$$

Waves entering on starboard side are indicated by ' + ' whereas waves entering on port side are indicated with a '-' and head sea corresponds to $\beta_{s}=180 \mathrm{deg}$.

Finally, the mean directional spread is given by

$$
\sigma_{s}=\left(2-\frac{2}{m_{0}} \sqrt{d^{2}+c^{2}}\right)^{0.5}
$$

which should not be confused with the spreading parameter $s$, since the directional spread $\sigma_{s}$ is measured in radians.

\section{Numerical Simulations}

This section presents results of the Bayesian modelling when the procedure is applied to stochastic numerical simulations of motion components. As it will be seen estimations are carried out with both one and two hyperparameters in the Bayesian modelling. This is in order to illustrate the problems encountered when only one hyperparameter is considered. It should be noted that theoretical aspects, related to the Bayesian modelling with only one hyperparameter, can be found in e.g. Iseki and Terada [14] and Nielsen [20]. 
4.1. Generation of Time Series. Ship motions can, in principle, be found by a time domain solution

$$
\boldsymbol{\eta}=\boldsymbol{\eta}(t)
$$

of the generalised equations of motion where the motions, in six degrees of freedom, $\eta_{i}, i=1,2, \ldots, 6$, are the surge, sway, heave, roll, pitch, and the yaw. In addition, global ship responses, e.g. the vertical acceleration and the wave induced bending moment, can be derived from the ship motions. In the following, a general global ship response $R(t)$ will be considered, without differentiation between ship motions and derived responses.

On the assumption of a linear relationship between responses and wave excitations, the time domain solution of the response $R(t)$ of a ship can be expressed in terms of the complex-valued frequency response function $\Phi_{R}(\omega, \beta)$, see for example St Denis and Pierson [6]. In this paper, the time domain solution of the response is, however, presented with the same format as in Jensen and Capul [15], although the latter reference considers only unidirectional waves. Thus, the response is written as a Gaussian process introduced by the set of uncorrelated, standard normal distributed variables $u_{m n}$ and $\bar{u}_{m n}$. Hence,

$$
R(t)=\sum_{n=1}^{N_{0}} \sum_{m=1}^{M_{0}}\left[u_{m n} c_{m n}(t)+\bar{u}_{m n} \bar{c}_{m n}(t)\right]
$$

The deterministic coefficients $c_{m n}(t)$ and $\bar{c}_{m n}(t)$ are given by

$$
\begin{gathered}
c_{m n}(t)=\sigma_{m n}\left|\Phi_{R}\left(\omega_{n}, \beta_{m}\right)\right| \cos \left(\omega_{e, n} t+\epsilon_{m n}\right) \\
\bar{c}_{m n}(t)=-\sigma_{m n}\left|\Phi_{R}\left(\omega_{n}, \beta_{m}\right)\right| \sin \left(\omega_{e, n} t+\epsilon_{m n}\right) \\
\sigma_{m n}^{2}=E\left(\omega_{n}, \beta_{m}\right) \Delta \omega_{n} \Delta \beta_{m}
\end{gathered}
$$

where it should be noted that the discretised number of wave frequencies $N_{0}$ and the discretised number of headings $M_{0}$ not (necessarily) take the same numbers as in the estimation analysis, cf. Eqs. (8) and (9). Furthermore, it should be realised that the variation over time is expressed in terms of the encounter frequency which is given by Eq. (2).

$E\left(\omega_{n}, \beta_{m}\right)$ is the directional wave spectrum under the assumption that the wave direction is measured relative to the ship course (i.e. $\beta=\theta$ ), and $\Delta \omega_{n}$ and $\Delta \beta_{m}$ are the increments of the discrete wave frequencies and the discrete headings, respectively. The phase angles are calculated from

$$
\epsilon_{m n}=\frac{\operatorname{Im}\left[\Phi_{\mathrm{R}}\left(\omega_{\mathrm{n}}, \beta_{\mathrm{m}}\right)\right]}{\operatorname{Re}\left[\Phi_{\mathrm{R}}\left(\omega_{\mathrm{n}}, \beta_{\mathrm{m}}\right)\right]}
$$

It should be noted that for an equidistant frequency discretisation, the signal $R(t)$ will repeat itself after a period of $\frac{2 \pi}{\Delta \omega}$. Thus, in order to avoid this problem, the frequency discretisation is taken to 
TABLE 1. Main dimensions of the considered ship.

\begin{tabular}{lr}
\hline Length, $L_{\mathrm{pp}}$ & $275.0 \mathrm{~m}$ \\
Breadth, $B_{\mathrm{mld}}$ & $40.0 \mathrm{~m}$ \\
Draught, $T$ & $12.0 \mathrm{~m}$ \\
Displacement & $50,000 \mathrm{t}$ \\
\hline
\end{tabular}

be non-equidistant

$$
\omega_{i+1}=\omega_{i}+c \cdot p_{i}
$$

with $c$ as an 'appropriately' small factor while $p_{i}$ denotes a stochastic variable with values between 0 and 1.

4.2. Setup of Test Cases. The numerical study will be carried out for a container vessel with characteristics as seen in Table 1. The complex-valued frequency response functions for the ship have been calculated by the 3-dimensional time domain code Wasim, e.g. DNV [7]. In the cases, which will be studied, three responses are considered. The responses are the sway, the heave and the pitch motions, and the speed of the vessel is $V=10.0 \mathrm{~m} / \mathrm{s}$ in all the numerical simulations. It should be realised that the sway response is an asymmetric response with respect to waves entering on the starboard/port side, cf. Nielsen [20] and Tannuri et al. [27].

The wave excitations, applied in the numerical simulation of the motion measurements, are based on a Pierson Moskowitz (PM) spectrum, including directional spreading. Hence, the bimodal spectrum given by Hogben and Cobb [11] is applied. The wave spectrum is introduced in two ways: In the first way, a unimodal PM wave spectrum is assumed, whereas a bimodal PM wave spectrum is considered in the second way. The test cases are summarised in Table 2, where it is seen that Case A corresponds to excitations characterised by swells with a significant wave height $H_{s}=2.0 \mathrm{~m}$, a peak period $T_{p}=14.0 \mathrm{~s}$, a spreading parameter $s=4.0$ and a mean wave heading $\beta=+105$ deg. Case B is a combination of wind sea and swells having an energy content represented by $H_{s}=3.0 \mathrm{~m}$ and $H_{s}=2.0 \mathrm{~m}$, respectively. The remaining wave parameters are given in Table 2 .

TABLE 2. The underlying wave parameters of the test cases and the corresponding speed of the vessel.

\begin{tabular}{cccccc}
\hline Case & $H_{s}[\mathrm{~m}]$ & $T_{p}[\mathrm{~s}]$ & $s$ & $\beta_{s}[\mathrm{deg}]$. & $V[\mathrm{~m} / \mathrm{s}]$ \\
\hline A & 2.0 & 14.0 & 4.0 & +105 & 10.0 \\
B & $3.0 / 2.0$ & $8.0 / 14.0$ & $3.0 / 4.0$ & $+015 /-135$ & 10.0 \\
\hline
\end{tabular}



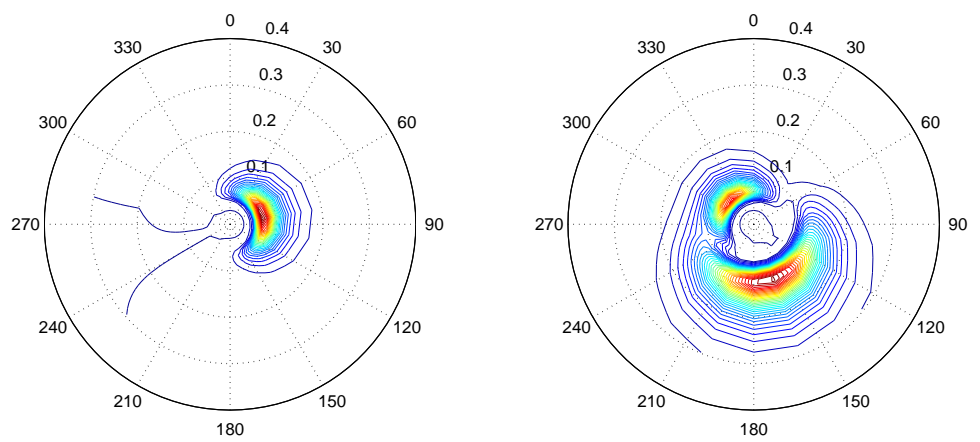

Figure 1. Contour plots of the directional wave spectra of Cases A (left) and B (right).

The directional wave spectra of Cases A and B are visualised in Figure 1 which shows contour plots of the wave spectra in a polar format. In the estimation procedure, the vessel is assumed to be at a course corresponding to $0 \mathrm{deg}$. in the polar plots. The directions are given in degrees and the wave frequencies are given in $\mathrm{Hz}$.

In the analysis, each of the cases listed in Table 2 is applied to simulate 20 runs of the set of responses (sway, heave, pitch) mentioned above. All the runs have a duration of 15 minutes corresponding to 900 seconds. Furthermore, it should be noted that no white noise has been added to the numerical generated time series and, similar, no uncertainties are considered in the estimations, so that the used complex-valued frequency response functions are assumed to give a perfect description of the hydrodynamic behaviour of the ship.

4.3. Minimum of ABIC. In accordance with theory, the best solution, i.e. the best estimate of the directional wave spectrum, to the observed data corresponds to the value of the hyperparameter(s) that gives the minimum value of ABIC. In case of only one hyperparameter in the Bayesian
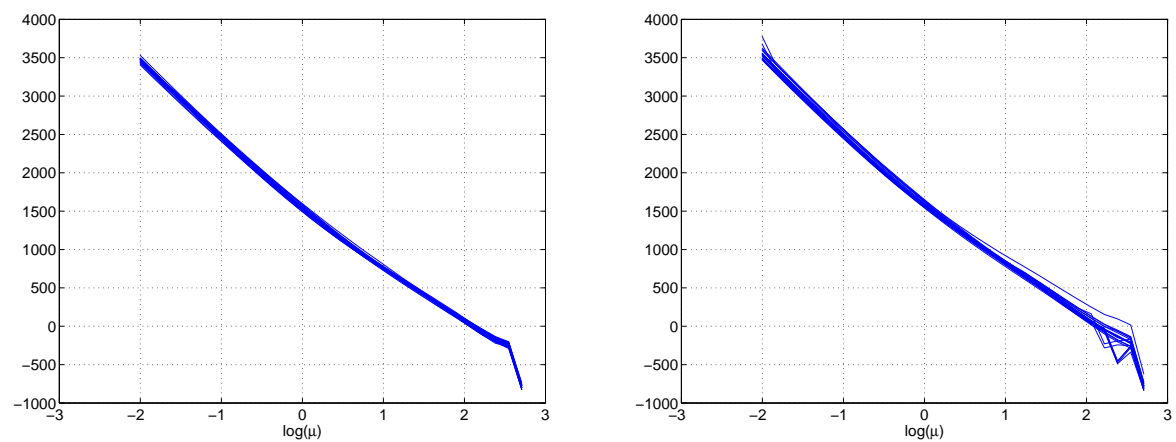

Figure 2. ABIC plotted for all twenty estimations in Cases A (left) and B (right) when only one hyperparameter is considered in the Bayesian modelling. 
modelling the results of ABIC are shown in Figure 2. Thus, ABIC is depicted as function of the logarithm to the hyperparameter $\mu$ in Cases $\mathrm{A}$ and $\mathrm{B}$, and the results of all twenty estimations in each case are seen in the plots. The hyperparameter is denoted by $\mu$ in the case of only one hyperparameter in order to illustrate that the hyperparameter represents another prior distribution than do $u$ and $v$ in the case of two hyperparameters.

From Figure 2 it is seen that ABIC decreases monotonously as $\mu$ increases; as a matter of fact, ABIC decreases infinitely as $\mu$ approaches infinity. The reason is that the prior information is described by only one hyperparameter although two sorts of prior distributions are considered. Hence, a problem similar to the improper formulation of ABIC, mentioned by Fukahata et al. [9], is observed. In the other direction of $\mu$, i.e. for $\mu \rightarrow 0$, ABIC increases until the solution stops converging. Figure 2 illustrates therefore, indeed, the problem in obtaining, automatically, the best solution, i.e. the best wave spectrum, to the observed data. Instead, the best solution must be chosen from a manual selection in the specific cases.

The introduction of two hyperparameters into Bayesian modelling was described in Section 2. Hence, each of the prior distributions is represented by its own corresponding hyperparameter and, therefore, the value of ABIC is given as a surface in the space of $(u, v)$. In Figure 3, the surfaces of ABIC are shown for one single estimation (out of twenty) in Cases A and B. From the figure it is seen that ABIC has a distinct minimum for a given set of $u$ and $v$ in Case A as well as Case B. Thus, the best solution can be obtained automatically; it is understood that the minimum value of ABIC does correspond to the best estimate of the wave spectrum.
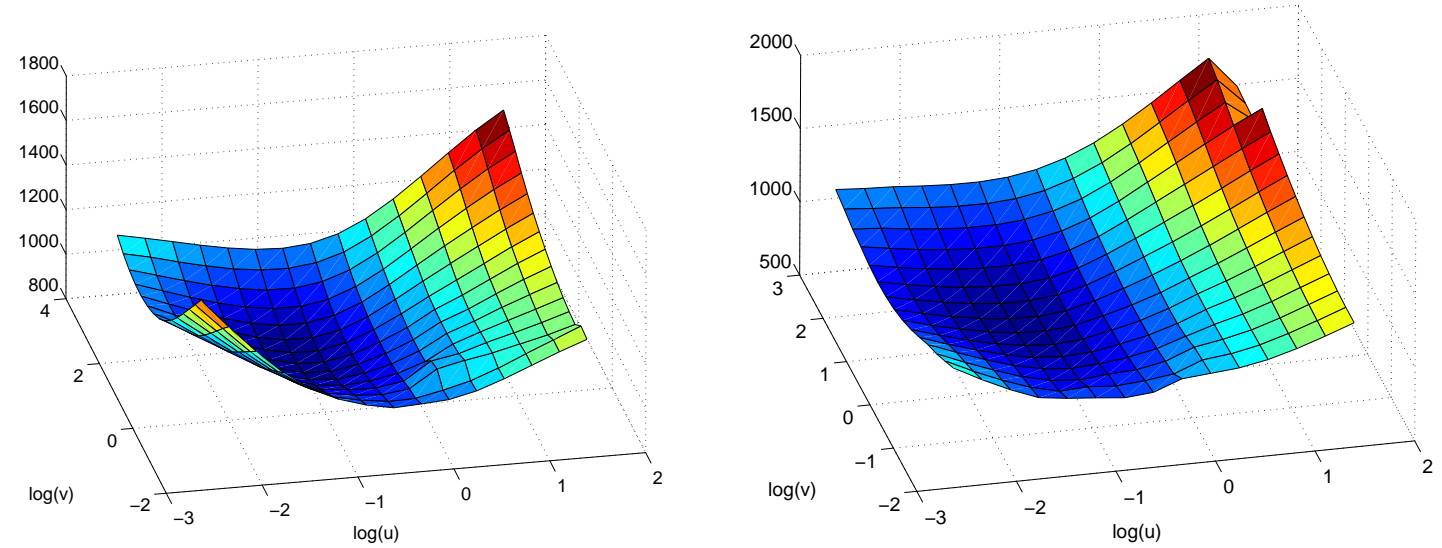

Figure 3. Examples of the ABIC surfaces in Case A (left) and Case B when two hyperparameters are considered in the Bayesian modelling. 
It should be noted that similar surfaces, with a unique minimum, are observed in all of the twenty estimations in each of the cases (A and B).

4.4. Estimation of Wave Spectra. In the preceding subsection it was illustrated that the Bayesian modelling did not give a unique minimum in the case with only one hyperparameter, which was in contrast to the case of two hyperparameters. A reasonable solution can, however, be obtained for the estimations based on one hyperparameter, since 'a best solution' can be selected manually, because the sought solution is known in advance. That is, the estimated wave spectrum with the best resemblance to the true (known) spectrum is - manually - selected. In the following, the results are, therefore, only selected automatically for the Bayesian modelling based on two hyperparameters.

The results of the estimation analyses are presented in Tables 3 and 4 . Thus, these tables list the characteristic wave parameters as calculated by Eqs. (16)-(23) and, specifically, the significant wave height $H_{s}$, the peak period $T_{p}$, the mean period $T_{s}$, the mean direction $\theta_{s}$, and the mean directional spread $\sigma_{s}$ are found in the tables. It should be noted that the shape parameter $\lambda$ of the wave spectrum ( $\lambda=1$ for a PM spectrum) is not included in the tables, since this parameter is difficult to obtain. A somewhat similar measure could be established by the combined measures of skewness and kurtosis of the wave spectrum. In the analysis, these numbers are, however, not dealt with. Finally, it should be mentioned that in the estimation analysis the wave field is discretised into $N=30$ wave frequencies $([0.01-0.30 \mathrm{~Hz}])$ and $M=18$ directions $([0-360 \mathrm{deg}]$.$) . The$ sensitivity to discretisation is not studied, but it should be noted that a previous study, Nielsen [20], indicates that the solution is not particularly sensitive to the values of $N$ and $M$ (if chosen appropriately), and in the study it was found that $N=30$ and $M=18$ are reasonable values to use in the discretisation of the wave field.

TABle 3. True and estimated wave parameters. Numbers for one hyperparameter (left) and two hyperparameters (right) are shown.

\begin{tabular}{|c|c|c|c|c|c|c|c|c|c|c|}
\hline \multicolumn{2}{|l|}{ Case } & $\begin{array}{l}H_{s} \\
{[\mathrm{~m}]} \\
\end{array}$ & \multicolumn{2}{|c|}{$\begin{array}{l}T_{p} \\
{[\mathrm{~s}]}\end{array}$} & \multicolumn{2}{|c|}{$\begin{array}{l}T_{s} \\
{[\mathrm{~s}]}\end{array}$} & \multicolumn{2}{|c|}{$\begin{array}{c}\theta_{s} \\
\text { [deg.] }\end{array}$} & \multicolumn{2}{|c|}{$\begin{array}{c}\sigma_{s} \\
{[\mathrm{deg} .]}\end{array}$} \\
\hline \multirow[t]{3}{*}{$\mathrm{A}$} & true & 2.0 & \multicolumn{2}{|c|}{14.0} & \multicolumn{2}{|c|}{11.0} & \multicolumn{2}{|c|}{+105} & \multicolumn{2}{|c|}{36} \\
\hline & mean & $1.9^{*} \quad 2.0$ & $13.1^{*}$ & 12.8 & $11.2^{*}$ & 11.1 & $+109^{*}$ & +109 & $46^{*}$ & 46 \\
\hline & error & $-5 \%^{*} \quad 0$ & $-6 \%^{*}$ & $-9 \%$ & $2 \%^{*}$ & $1 \%$ & $4^{*}$ & 4 & $10^{*}$ & 10 \\
\hline \multirow[t]{3}{*}{ B } & true & 3.6 & \multicolumn{2}{|c|}{-} & \multicolumn{2}{|c|}{7.5} & \multicolumn{2}{|c|}{-172} & \multicolumn{2}{|c|}{-} \\
\hline & mean & $3.2^{*} \quad 3.3$ & - & - & $8.2^{*}$ & 8.2 & $-156^{*}$ & -155 & - & - \\
\hline & error & $-11 \%^{*}$ & - & - & $9 \%^{*}$ & $9 \%$ & $16^{*}$ & 17 & - & - \\
\hline
\end{tabular}

${ }^{*}$ Value based on manual selection of the hyperparameter. 
TABle 4. Partitioning of the bimodal wave spectrum. Mean values from the twenty simulations/estimations are shown.

\begin{tabular}{|c|c|c|c|c|c|c|c|c|c|c|c|}
\hline \multirow[b]{2}{*}{ Case } & & \multicolumn{5}{|c|}{ Wind sea } & \multicolumn{5}{|c|}{ Swells } \\
\hline & & $\begin{array}{l}H_{s} \\
{[\mathrm{~m}]}\end{array}$ & $\begin{array}{l}T_{p} \\
{[\mathrm{~s}]}\end{array}$ & $\begin{array}{l}T_{s} \\
{[\mathrm{~s}]}\end{array}$ & $\begin{array}{c}\theta_{s} \\
{[\mathrm{deg} .]}\end{array}$ & $\left.\begin{array}{c}\sigma_{s} \\
{[\mathrm{deg} .}\end{array}\right]$ & $\begin{array}{l}H_{s} \\
{[\mathrm{~m}]}\end{array}$ & $\begin{array}{l}T_{p} \\
{[\mathrm{~s}]}\end{array}$ & $\begin{array}{l}T_{s} \\
{[\mathrm{~s}]}\end{array}$ & $\begin{array}{c}\theta_{s} \\
\text { [deg.] }\end{array}$ & $\begin{array}{c}\sigma_{s} \\
{[\mathrm{deg} .]}\end{array}$ \\
\hline \multirow[t]{3}{*}{ B } & true & 3.0 & 8.0 & 6.5 & +015 & 41 & 2.0 & 14.0 & 11.0 & -135 & 36 \\
\hline & bay $^{1}$ & $2.6^{*}$ & $7.2^{*}$ & $7.4^{*}$ & $+002^{*}$ & $29^{*}$ & $1.8^{*}$ & $14.1^{*}$ & $14.0^{*}$ & $-125^{*}$ & $22^{*}$ \\
\hline & bay $^{2}$ & 2.7 & 7.4 & 7.4 & +004 & 45 & 1.9 & 14.6 & 14.1 & -125 & 31 \\
\hline
\end{tabular}

It is seen that Table 3 contains results for only the significant wave height, the mean wave period and the mean wave direction for Case B, since the table represents the total wave system. In addition to Table 3, Table 4 needs therefore to be considered for the partitioning, e.g. Komen et al. [16] and Gerling [10], of the wave spectrum in Case B.

From Table 3, it can be seen that three values, denoted 'true', 'mean' and 'error', are given to each case. The values 'true' correspond to the exact parameters of the wave spectrum as seen from Table 2. For the two other values - 'mean' and 'error' - two numbers are given to each wave parameter. The first number, i.e. the left one, yields the result as obtained by the Bayesian modelling based on only one hyperparameter, whereas the second number, i.e. the right one, yields the result as obtained by the Bayesian modelling with two hyperparameters. It is understood that the value 'mean' represents the mean value of the specific estimated parameter for the twenty simulations/estimations carried out in the individual cases. The value 'error', which is also based on the mean of the twenty estimations, yields the error between the estimated mean and the true value relative to the true value, except for the parameters related to the wave direction where the absolute value of the error is shown.

Tables 3 and 4 show that the results of the Bayesian modelling match the true wave parameters with reasonable accuracies. It is seen that the energy content, represented by the significant wave height, is slightly underestimated in Case B. This problem originates probably because of filtering, since it is difficult to capture the high-frequency excitations due to the relatively large inertia of the considered vessel, cf. Table 1. The problem is, to some extent, visualised in Figure 4 which shows the distribution of energy with frequency in the form of the frequency wave spectra. Results for both one hyperparameter (left plots) and two hyperparameters (right plots) are shown, and the results of all twenty estimations is visualised in each of the cases. 
Based on the plots of Case B, it is seen that the energy distribution of the wind sea component of the wave spectrum does not follow the true distribution exactly. Hence, the location of the estimated (wind) peak is shifted and the energy is contained within a shorter frequency range. The problems related to filtering will, however, not be discussed any further here. Instead, reference is given to Nielsen [21] that deals with the problem in detail.

Examples on the estimated directional wave spectra in Case B are visualised in Figure 5 which shows contour plots of the wave spectrum in a polar format. It should be pointed out that the figure illustrates only one of the twenty estimated directional wave spectra, although similar plots are observed in the remaining cases. In the figure, the true directional spectrum is shown to the left and the results based on only one and on two hyperparameters are shown in the middle and to the right, respectively. The vessel has a course corresponding to 0 deg., and the waves are depicted as approaching.

In summary on the results from the Bayesian modelling it is seen that the directional wave spectrum is estimated with a reasonable accuracy compared to the true spectrum. It is, however, important to emphasise that it is only the results based on the Bayesian modelling with two hyperparameters that are determined automatically. The results based on one hyperparameter are, on the other hand, selected manually, since the evaluation of ABIC does not suggest an optimal value for the hyperparameter because no unique minimum is observed for ABIC. In this respect, it should be kept in mind that a manual selection of the 'best solution' is only possible since the sought - true wave spectrum is known in advance.
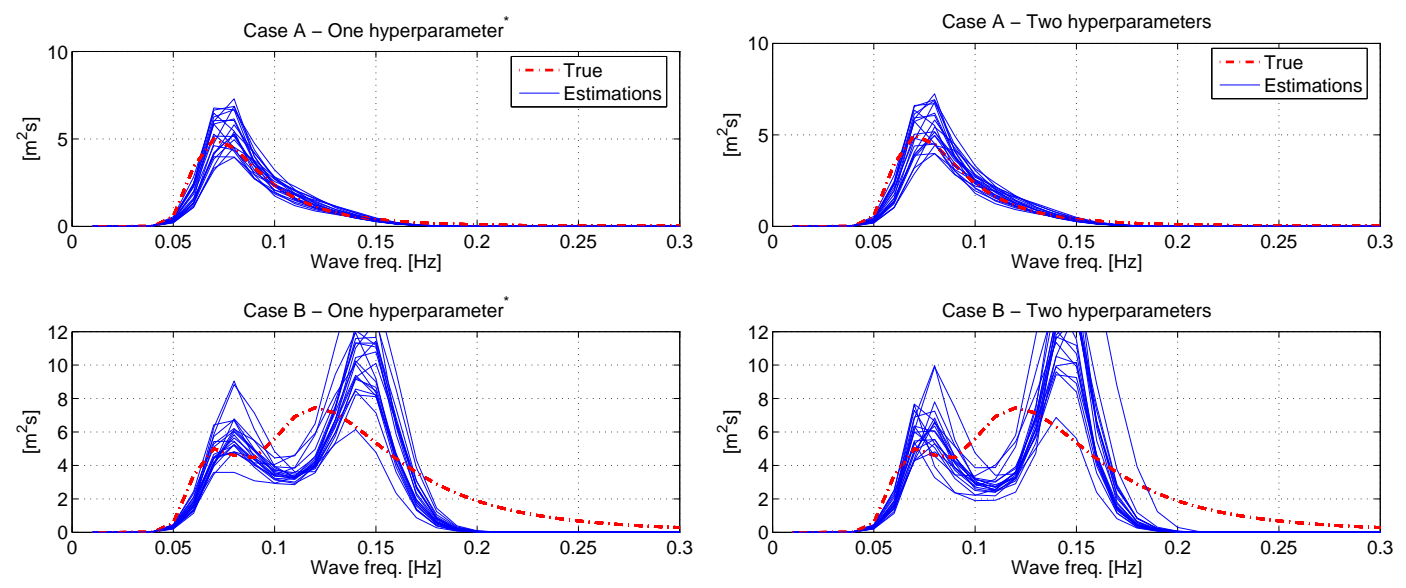

Figure 4. The estimated frequency wave spectra of Cases $A$ and B. All twenty estimations are shown in each of the plots. 

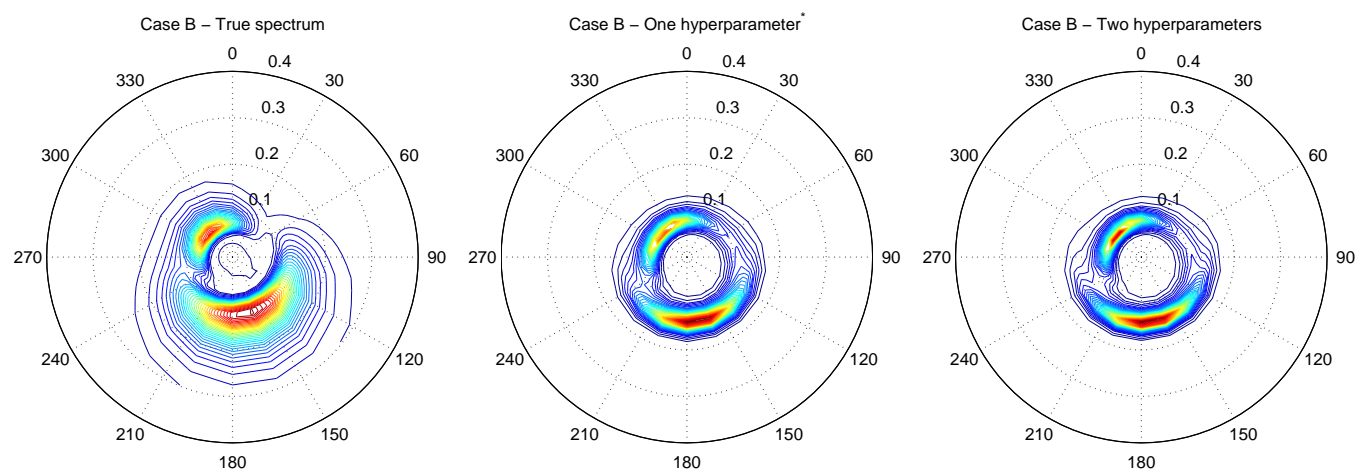

FiguRE 5. Examples of contour plots of the estimated directional wave spectra in

Case B.

For comparative reasons, as regards the Bayesian modelling based on two hyperparameters, it should be mentioned that by picking a set of hyperparameters $(u, v)$, which does not give the minimum value of $\mathrm{ABIC}$, both the content of energy and the specific distribution of energy are estimated worse than seen for the optimal set $(u, v)_{\min }$ of the hyperparameters.

\section{Analysis of Full-scale Measurements}

Based on full-scale motion measurements recorded on the vessel studied in the previous section, see Table 1, wave spectra have been estimated. The data consists of eight sets: A, B, ..., H, each of a duration of 15 minutes. The duration is taken in the middle of a 30 minutes period used for the wave radar (WAVEX) estimations and during this time, the operational conditions were (nearly) constant. The estimations are based on the responses of heave, roll and pitch. For a thorough discussion on the type of responses on which the estimations should be based, reference is given to e.g. Tannuri et al. [27], Aschehoug [3] and Nielsen [20]. In the estimation analyses it is, however, important to emphasise that the complex-valued transfer functions are believed to give a perfect relationship between the wave excitations and the ship responses. This means that the natural uncertainty related to the transfer functions is not accounted for. This includes phenomena such as: not perfectly controlled/known operational conditions (in particular, as regards the loading condition); unknown noise phenomena in the response signals; the degree of non-linearity between excitations and responses is difficult to predict about; relative to the size of the ship the high-frequency wave components induce no significant motions; etc. Consequently, the estimated directional wave spectrum and the associated underlying wave parameters carry uncertainty which is, however, not considered in the Bayesian modelling. Similarly, sensitivity analyses of the transfer functions as regards the final outcome of the wave estimations have not been conducted. 
TABLE 5. Comparison of wave parameters; the significant wave height $H_{s}$, the mean wave period $T_{s}$ and the mean wave heading $\beta_{s}$.

\begin{tabular}{|c|c|c|c|c|c|c|c|c|c|}
\hline \multirow[b]{2}{*}{ Data } & \multicolumn{3}{|c|}{$H_{s}[\mathrm{~m}]$} & \multicolumn{3}{|c|}{$T_{s}[\mathrm{~s}]$} & \multicolumn{3}{|c|}{$\beta_{s}[$ deg.] } \\
\hline & bay & sat & wav & bay & sat & wav & bay & sat & wav \\
\hline A & 2.7 & 3.0 & $3.0( \pm 0.4)$ & 7.9 & 8.1 & 6.9 & -090 & -040 & -100 \\
\hline B & 2.7 & 3.0 & $2.5( \pm 0.3)$ & 7.6 & 7.9 & 6.8 & -060 & -045 & -045 \\
\hline $\mathrm{C}$ & 2.0 & 2.7 & $2.6( \pm 0.3)$ & 7.6 & 7.4 & 6.5 & -060 & -030 & -060 \\
\hline $\mathrm{D}$ & 1.5 & 2.3 & $2.5( \pm 0.3)$ & 10.7 & 6.6 & 6.6 & +015 & -160 & +065 \\
\hline $\mathrm{E}$ & 4.6 & 3.0 & $4.9( \pm 1.5)$ & 9.3 & 9.5 & 9.2 & +170 & +160 & -150 \\
\hline $\mathrm{F}$ & 3.8 & 5.6 & $4.8( \pm 1.0)$ & 11.1 & 10.6 & 8.6 & +020 & +040 & 000 \\
\hline G & 4.2 & 4.2 & $4.5( \pm 0.6)$ & 11.0 & 11.1 & 8.7 & +135 & +125 & +120 \\
\hline $\mathrm{H}$ & 4.6 & 5.2 & $4.4( \pm 1.4)$ & 9.3 & 10.5 & 8.8 & +130 & +115 & +145 \\
\hline
\end{tabular}

5.1. Wave Parameters and Frequency Wave Spectra. The results of the estimations are shown in Table 5. The table lists values of the significant wave height $H_{s}$, the mean wave period $T_{s}$ and the mean heading $\beta_{s}$ relative to the waves; with all the individual parameters calculated according to Eqs. (16)-(23). The table shows results by the Bayesian modelling (bay), by the wave radar system WAVEX (wav), e.g. MIROS [17], and by satellite measurements (sat), e.g. Oceanweather [22]. As regards WAVEX, the technical manual [17] considers the statistical uncertainty of the measurements related to the significant wave height and this uncertainty is included in parenthesis in Table 5. It should be emphasised that all results of the Bayesian modelling are based on the optimum values of the hyperparameters, which are the pairs of $u$ and $v$ that give minimum ABIC for the specific data sets.

From Table 5 it is seen that the energy content, represented by the significant wave height, is estimated with a reasonable agreement between the three different estimations. The exact distribution of energy with frequency can be seen in Figure 6 which shows the frequency wave spectra of all eight cases. In the figure, results are shown for WAVEX and for the Bayesian Method. It is not possible to obtain frequency wave spectra from the satellite measurements. Figure 6 seems to suggest that the best frequency-wise agreement between WAVEX and Bayesian modelling, in general, is found in the lower frequency region. In particular, with focus on Data D, it is observed that the Bayesian Method captures only a swell part of the wave spectrum, whereas WAVEX estimates a similar swell part (however with less energy) and, in addition, a wind generated peak. This fact has probably to do with filtering introduced because of the relatively large inertia of the ship, which means that high-frequency components of the wave spectrum is not estimated as accurately as low-frequency ones by the Bayesian Method. This phenomenon is a general problem for response-based estimation 
of wave spectra and has been reported by e.g. Tannuri et al. [27], Pascoal et al. [23] and Nielsen $[21]$.

The visualisations of the wave spectra, Figure 6, illustrate also the differences between WAVEX and the Bayesian Method observed in Table 5 on the mean wave period. At this point, it should, however, be mentioned that the best agreement on $T_{s}$ is actually found between the satellite measurements and the results of the Bayesian modelling (except Data D). As regards the mean wave heading $\beta_{s}$, Table 5 reveals no clear tendency between the results, since the agreement between the three different estimations seems fair. The exception is, however, Data D, which has already been discussed and, furthermore, it is noted that WAVEX estimates waves entering on the port side of the ship for Data E, contrary to the results of the Bayesian modelling and the satellite measurements, which both estimate waves entering on the starboard side.
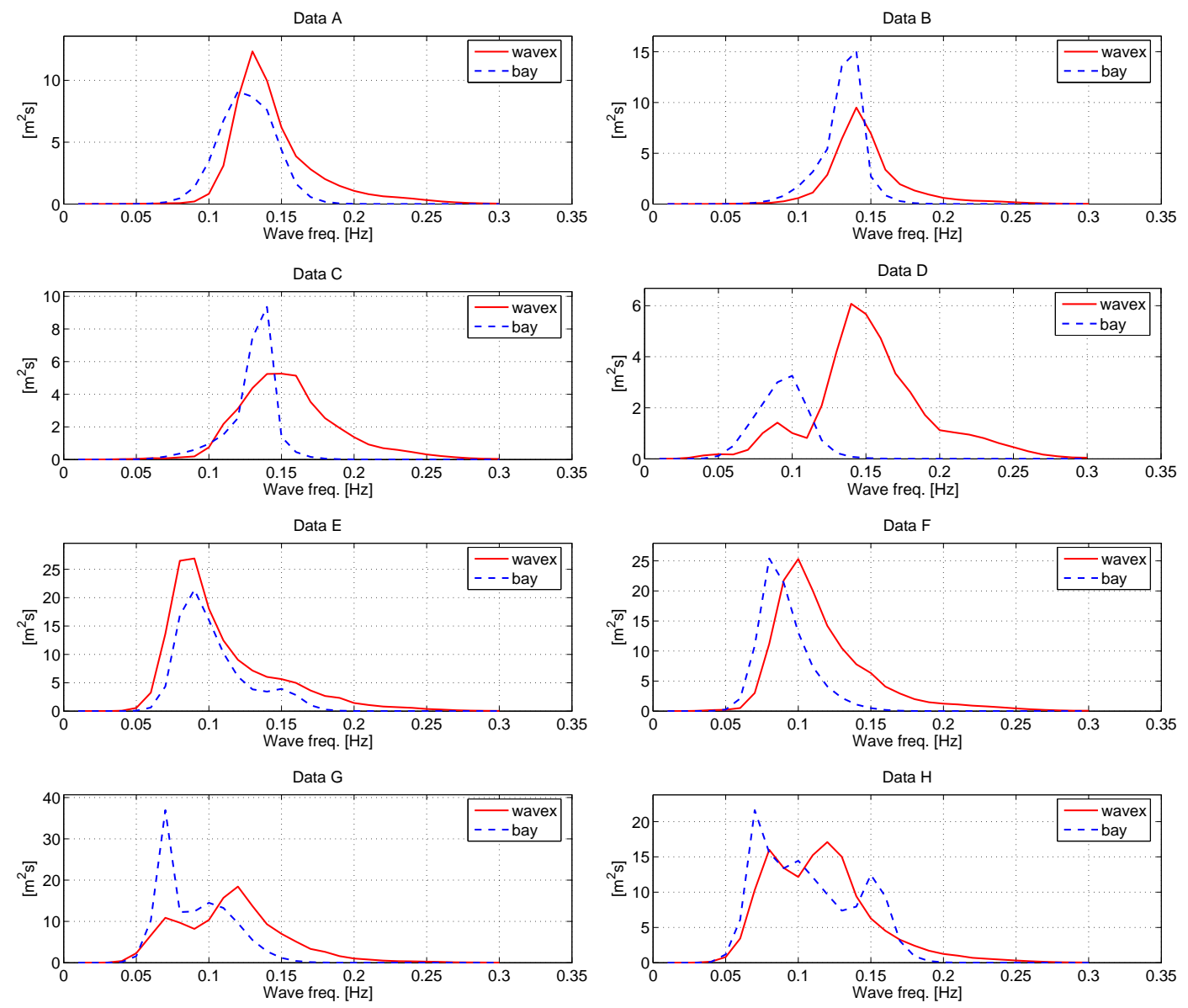

FiguRE 6 . The estimated frequency wave spectra. (Note the difference in scales.) 
As a final comment on the results it should be kept in mind that the three different means of estimation all give an estimate of the true wave spectrum, so that it cannot be decided which one is to be taken to be the truth. Overall, the resemblance is, however, fair.

\section{Summary And Conclusions}

In the paper, two hyperparameters were introduced into Bayesian modelling based on two prior distributions. The introduction is necessary in order to properly control the amount of smoothing applied in the directional as well as the frequency ranges as regards the good-fit versus the stability of the solution. The optimal amount of smoothing, expressed in terms of two hyperparameters, is determined by use of ABIC (a Bayesian Information Criterion). In the literature, traditionally, only one hyperparameter has been applied to describe the directional- and frequency-wise smoothing of the wave spectrum. ABIC has therefore been rewritten so that a proper formulation has been obtained.

From numerical simulations of motion measurements with exact known underlying wave parameters it was seen that the best solution was obtained for values of the hyperparameters corresponding to the minimum of ABIC. Hence, the analysis of the associated estimations of the wave spectrum revealed that the best solution of the Bayesian modelling did predict the wave parameters with reasonable accuracy. This was not the case for the Bayesian modelling when only one hyperparameter was taken into account to model the prior distributions, since a unique minimum for ABIC was not observed in this specific case. Thus, the best solution had to be selected manually when only one hyperparameter was considered, and a manual selection was only possible because the true wave spectrum was known in advance.

In addition, the paper considered full-scale motion measurements and from analyses it was seen that the estimated wave spectra agreed reasonably well with results by the wave radar system WAVEX and with ocean surface measurements obtained by satellite.

\section{AcKnowledgement}

The complex-valued frequency response functions and the full-scale motion measurements have been provided by Det Norske Veritas (DNV). In this relation, the Network of Excellence, MARSTRUCT, should be acknowledged for the sponsorship of a one-week stay at DNV. Finally, the inspiring discussions with Professor Jørgen Juncher Jensen is highly appreciated. 


\section{REFERENCES}

1. ADOPT, Advanced Decision Support System for Ship Design, Operation and Training, The EU project belongs to the Sixth Framework Programme. See the project web site http://adopt.rtdproject.net.

2. H. Akaike, Likelihood and Bayes Procedure, Bayesian Statistics (J. M. Bernado, M. H. De Groot, D. U. Lindley, and A. F. M. Smith, eds.), University Press, Valencia, 1980, pp. 143-166.

3. M. Aschehoug, Scientific Paper on The Sea State Estimation Methodology, Tech. report, SIREHNA, France, 2003, (Paper prepared in the HullMon+ project.).

4. A.C. Davison, Approximate Predictive Likelihood, Biometrika 73 (1986), 323-332.

5. F.W. Debord and B. Hennessy, Development of a Generalized Onboard Response Monitoring System, Tech. report, Ship Structure Committee, 1990, Report SSC-349.

6. M. St. Denis and W.J. Pierson, On the Motion of Ships in Confused Seas, Trans. SNAME 61 (1953).

7. DNV, User Manual: Wasim, 2005, Technical documentation by Det Norske Veritas (DNV).

8. Y. Fukahata, A. Nishitani, and M. Matsu'ura, Geodetic data inversion using ABIC to estimate slip history during one earthquake cycle with viscoelastic slip-response functions, Geophys. J. Int. 156 (2004), 140-53.

9. Y. Fukahata, Y. Yagi, and M. Matsu'ura, Waveform inversion for seismic source processes using ABIC with two sorts of prior constraints: Comparison between proper and improper formulations, Geophysical Research Letters 30 (2003), 38.1-38.4.

10. T.W. Gerling, Partitioning sequences and arrays of directional wave spectra into component wave systems, J. Atmos. Oceanic Technology 9 (1992), 111-136.

11. N. Hogben and F.C. Cobb, Parametric Modelling of Directional Wave Spectra, Proceedings of 18th Offshore Technology Conference (Houston, Texas), 1986.

12. M. Huss and A. Olander, Theoretical Seakeeping Predictions On Board Ships - A System for Operational Guidance and Real Time Surveillance, Tech. report, Naval Architecture, Department of Vehicle Engineering, Royal Institute of Technology, 1994.

13. T. Iseki and K. Ohtsu, Bayesian Estimation of Directional Wave Spectra Based on Ship Motions, Control Engineering Practice 8 (2000), 215-219.

14. T. Iseki and D. Terada, Bayesian Estimation of Directional Wave Spectra for Ship Guidance Systems, International Journal of Offshore and Polar Engineering 12 (2002), 25-30.

15. J.J. Jensen and J. Capul, Extreme Response Predictions for Jack-up Units in Second Order Stochastic Waves by FORM, Probabilistic Engineering Mechanics 21 (2006), no. 4, 330-338.

16. G.J. Komen, L. Cavaleri, M. Donelan, K. Hasselmann, S. Hasselmann, and P.A.E.M Janssen, Dynamics and modelling of ocean waves, Cambridge University Press, 1994.

17. MIROS, WAVEX - Principles of Operation, 1998, Technical documentation by MIROS.

18. J.K. Nielsen, N.H. Pedersen, J. Michelsen, U.D. Nielsen, J. Baatrup, J.J. Jensen, and E.S. Petersen, SeaSense Real-time Onboard Decision Support, Proceedings of WMTC2006 (London, UK), 2006.

19. U.D. Nielsen, Estimation of Directional Wave Spectra from Measured Ship Responses, Ph.D. thesis, Section of Coastal, Maritime and Structural Engineering, Department of Mechanical Engineering, Technical University of Denmark, May 2005. 
20. , Estimations of On-site Directional Wave Spectra from Measured Ship Responses, Marine Structures 19 (2006), no. 1, 33-69.

21. , Response-based Estimation of Sea State Parameters - Influence of Filtering, Ocean Engineering 34 (2007), 1797-1810.

22. Oceanweather, Oceanweather, Inc., 2005, http://www.oceanweather.com.

23. R. Pascoal, C.G. Soares, and A.J. Sørensen, Ocean Wave Spectral Estimation Using Vessel Wave Frequency Motions, Proceedings of OMAE2005 (Halkidiki, Greece), 2005.

24. W.H. Press, B.P. Flannery, S.A. Teukolsky, and W.T. Vetterling, Numerical Recipes in FORTRAN7r: The Art of Scientific Computing, 2nd ed., Cambridge University Press, 1992.

25. Z. Shun and P. McCullagh, Laplace Approximation of High Dimensional Integrals, Journal of Royal Statistical Society 57 (1995), 749-760.

26. S.B. Slaughter, M.C. Cheung, D. Sucharski, and B. Cowper, State of the Art in Hull Monitoring Systems, Tech. report, Ship Structure Committee, 1997, Report SSC-401.

27. E.A. Tannuri, J.V. Sparano, A.N. Simos, and J.J. Da Cruz, Estimating Directional Wave Spectrum Based on Stationary Ship Motion Measurements, Applied Ocean Research 25 (2003), 243-261. 